\title{
Spatial Dependence of Stimulus Competition in the Avian Nucleus Isthmi Pars Magnocellularis
}

\author{
Hannah M. Schryver ${ }^{a}$ Shreesh P. Mysore ${ }^{a, b}$ \\ ${ }^{a}$ Department of Psychological and Brain Sciences, Johns Hopkins University, Baltimore, MD, USA; \\ ${ }^{b}$ The Solomon H. Snyder Department of Neuroscience, Johns Hopkins University, Baltimore, MD, USA
}

\section{Keywords}

Sensory systems · Tectum · Birds · Electrophysiology ·

Inhibition · Stimulus selection

\begin{abstract}
The nucleus isthmi pars magnocellularis (Imc) is a group of specialized inhibitory neurons in the midbrain tegmentum, thought to be conserved across vertebrate classes. Past anatomical work in reptiles has suggested a role for it in stimulus selection, which has been supported by recent studies in avians. Additionally, focal inactivation of Imc neurons is known to abolish all competitive interactions in the optic tectum (OT; SC in mammals), a midbrain sensorimotor hub that is critical for the control of spatial attention, thereby revealing a key role for Imc in stimulus selection. However, the functional properties of Imc neurons are not well understood. Here, with electrophysiological experiments in the barn owl Imc, we show that Imc neurons themselves exhibit signatures of stimulus competition. Distant competing stimuli outside the spatial receptive field (RF) suppressed powerfully, and divisively, the responses of Imc neurons to stimuli inside the RF, and did so from all tested locations along the elevation as well as azimuth. Notably, this held true even for
\end{abstract}

(c) 2019 S. Karger AG, Basel

E-Mail karger@karger.com www.karger.com/bbe locations encoded by the opposite side of the brain from the one containing the recording site. This global divisive inhibition operated independently of the sensory modality of the competing stimulus. Thus, the Imc not only supplies inhibition to the OT to support competition there, but may itself be an active site of stimulus competition. These results from experiments in the barn owl shed light on the functional properties of a vital node in the vertebrate midbrain selection network.

(c) 2019 S. Karger AG, Basel

\section{Introduction}

In complex sensory environments animals must quickly select the most important stimulus location to guide adaptive behaviors such as mate identification, predator avoidance, foraging for food, and spatial navigation. Such selection depends both on the physical salience and behavioral relevance of stimuli in order to identify the spatial locus of highest priority [Fecteau and Munoz, 2006]. Forebrain as well as midbrain circuits have been identified as critical in the control of such spatial selection [Bisley and Goldberg, 2010; Krauzlis et al., 2013; Squire et 
al., 2013]. In this context, the role of a midbrain selection network, thought to be conserved across all vertebrate species, has been elaborated in some detail [Knudsen, 2011; Mysore and Knudsen, 2011b].

The midbrain selection network includes the superior colliculus (SC; called optic tectum, OT, in non-mammals) and key satellite structures, referred to as the isthmic nuclei. The SC/OT, specifically the intermediate and deep layers (called the SCid/OTid), encode stimulus salience and behavioral relevance in a topographic map of sensorimotor space [Knudsen, 2011; Krauzlis et al., 2013]. Experiments in behaving monkeys have demonstrated that the SCid is required for the selection of the target of spatial attention in the presence of competing distracters [McPeek and Keller, 2004; Lovejoy and Krauzlis, 2010].

In parallel, the presence of competitive interactions in the SCid/OTid is well established [Rizzolatti et al., 1974; Frost, 1978; Mysore et al., 2010]. Specifically, studies in the avian midbrain have shed light on how representations of competing stimuli in the SCid/OTid, which are instantiated as competitively suppressed neural responses, are produced. It is now known that inhibition from a GABAergic midbrain nucleus called isthmi pars magnocellularis (Imc) [Sereno and Ulinski, 1987; Wang et al., 2004] drives competitive inhibition and stimulus competition in the OTid. The Imc has a specialized pattern of antitopographic connectivity with the OT, sending widespread projections across the OT space map [Wang et al., 2004]. Notably, inactivation of the Imc abolishes all competitive inhibition in the OT [Marin et al., 2007; Mysore and Knudsen, 2013]. Together, this work across species has inferred a vital role for the Imc in controlling competitive representations in the SC/OT, thereby potentially driving spatial selection behavior.

Despite the importance of the Imc to spatial selection, and a detailed understanding of its encoding of visual space [Wang and Frost, 1991; Mahajan and Mysore, 2018], how stimulus competition is encoded within the Imc is largely unclear. An important property in this context is the ability for competitive interactions to operate across space, allowing stimuli present at nearby as well as distant spatial locations to influence one another's neural responses. Here, we examine whether and how Imc neurons respond to competing stimuli, and investigate the spatial dependence of stimulus competition within the Imc. Specifically, we ask how the responses of Imc neurons to a stimulus inside their receptive fields (RF) are modulated by the presence of a second competing stimulus located outside (or inside) the RF.

\section{Materials and Methods}

\section{Animals}

We performed electrophysiological recordings in 8 head-fixed adult barn owls (Tyto alba). Both male and female owls were used, and the birds were shared across different studies. All procedures for animal care and use were performed following approval by the Johns Hopkins University Institutional Animal Care and Use Committee, and in accordance with NIH guidelines for care and use of laboratory animals. Owls were group housed in an aviary with a 12/12-h light/dark cycle.

\section{Neurophysiology and Imc Targeting}

Experiments were performed following protocols that have been described previously [Mysore et al., 2010; Mysore and Knudsen, 2013; Mahajan and Mysore, 2018]. On experiment days, owls were anesthetized with isoflurane (2\%) and a mixture of nitrous oxide and oxygen (45:55) and wrapped in a flexible jacket. The birds were then head-fixed in a sound-attenuating booth. Isoflurane was turned off immediately after the bird was secured and was not turned back on for the remainder of the experiment. In some cases, nitrous oxide was also turned off $30 \mathrm{~min}$ before the recording session. As recovery from isofluorane occurs well under $30 \mathrm{~min}$ after it is turned off, and recovery from nitrous oxide occurs within a minute, recordings were made in animals that were not anesthetized, and in some cases, non-tranquilized. Previous work has demonstrated that neural responses in the midbrain network to stimulus competition protocols do not differ between nitrous oxide tranquilized versus non-tranquilized conditions [Mysore et al., 2011]

Extracellular activity was recorded from Imc sites using epoxycoated tungsten microelectrodes of high impedance (A-M Systems, $5 \mathrm{M} \Omega$ at $1 \mathrm{kHz}, 250 \mu \mathrm{m}$ shaft diameter). All dorsoventral penetrations targeting the Imc were made at an angle of $5^{\circ}$ from the vertical (medial-leading direction) to avoid a major blood vessel in the path to the Imc. Spike times were recorded using TuckerDavis hardware and custom MATLAB software.

The Imc is an oblong structure in the owl midbrain that is elongated along the rostrocaudal axis, and that lies parallel to the rostrocaudal axis of the OT. It is approximately $2.8 \mathrm{~mm}$ long rostrocaudally and $0.35 \mathrm{~mm}$ tall dorsoventrally, and appears as a $700 \times$ $350-\mu \mathrm{m}$ elliptical disk in coronal sections. It is located approximately $16 \mathrm{~mm}$ ventral to the surface of the brain, and approximately $500 \mu \mathrm{m}$ medial to the medial-most part of the OT. In previous work, we have confirmed in vivo targeting of the Imc with fluorescent dye injection [Mysore and Knudsen, 2013], and with electrolytic lesions [Mahajan and Mysore, 2018]. The Imc was targeted in our experiments using either established procedures from those studies, or by referencing rostral-caudal and lateral-medial coordinates from prior successful targeting experiments in a given bird. Briefly, to target Imc de novo, we first navigated to the superficial layers in the ventral portion of the OT that encoded for around spatial locations between $\mathrm{C} 30^{\circ}$ and $\mathrm{C} 40^{\circ}$ in the azimuth, and $-35^{\circ}$ to $-40^{\circ}$ in elevation using established OT targeting procedures [Knudsen, 1982], and then jumped medially by approximately $800 \mu \mathrm{m}$ from there. Imc neurons have characteristic electrophysiological signatures in that they typically respond with large (triphasic) spikes to visual stimuli across a broad range of elevations spanning both upper and lower elevations. By contrast, nearby areas such as the OT and the isthmi pars parvocellularis 
(Ipc) respond to restricted portions of visual space typically centered in either the upper or the lower elevation, and have different response properties [Knudsen, 1982; Asadollahi et al., 2010]. Once in the Imc, we moved the electrode ventrally in 5- to $10-\mu \mathrm{m}$ steps to isolate single units. Often, more than one unit waveform exceeded the threshold, resulting in a multiunit recording site. Our recordings, therefore, consisted of a mix of well-isolated single units as well as multiunit sites; the ratio of single to multiunit sites is $18: 31$ in our dataset.

\section{Stimuli}

Our experiments involved presenting either one stimulus or two stimuli simultaneously to the owl. Throughout the paper, S1 refers to the stimulus presented inside the spatial RF of the recording site, and it was always a visual stimulus. S2 refers to the second stimulus, presented simultaneously with S1, either outside or inside the RF. It was either a visual stimulus or an auditory noise burst stimulus. When S2's location was varied along the azimuth, it was presented typically at 10 locations outside the RF and 2 locations inside the RF each site. When S2's location was varied along the elevation, it was presented typically at 4 locations outside the $\mathrm{RF}$ and 7 locations inside. All stimuli were presented for $250 \mathrm{~ms}$ with an inter-stimulus interval of 1.5-3 s at all sampled locations. Every single or paired stimulus presentation was repeated 10-15 times at different location combinations (azimuth/elevation; inside RF/outside RF) and under different stimulus conditions (visual/auditory).

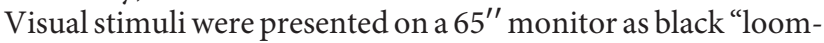
ing" stimuli upon a gray background at a fixed contrast [Mysore et al., 2010]; stimulus locations were defined in double pole coordinates relative to the midsagittal plane for the azimuth or the visual plane for elevation. Looming visual stimuli consisted of a black dot on a gray background that progressively increased in size over the period of stimulus presentation $(250 \mathrm{~ms})$ starting from a size of $0.6^{\circ}$ radius. The center of a looming stimulus remained constant throughout the duration of the motion stimulus (unlike translationally moving stimuli). The rate of change of the angular size of the dot, $d \Phi / d t$, where $\Phi$ is the visual half-angle subtended by the object at the eye, was held constant over the duration of stimulus presentation $(d \Phi / d t=\lambda)$, leading to a linear relationship between dot size and time. The constant $\lambda$ represented the loom speed of the stimulus in degrees per second. Loom speed was varied by changing the final size of the dot, while keeping the initial size $\left(0.6^{\circ}\right)$ and the duration of presentation $(250 \mathrm{~ms})$ fixed. Looming stimuli with a non-linear relationship of dot-size as a function of time (mimicking a constant approach speed) were not used for data collection because: (a) at moderately high approach speeds, these stimuli resulted in final dot sizes that were very large, making the study of stimulus integration within the visual RF difficult, and (b) such stimuli cause most of the spiking responses to occur over a very short window, making it difficult both to assess responses, and to assess changes to them [Mysore et al., 2010].

Looming dots evoke stronger responses in owl isthmotectal neurons than other kinds of stimuli tested (stationary dots, moving dots, broadband auditory bursts, etc.), and are more resistant to adaptation effects (which are encountered when performing repeated measurements for improving statistical power) [Asadollahi et al., 2010; Mysore et al., 2010]. Indeed, by virtue of resembling approaching objects, they are behaviorally highly salient. For these reasons, they are an excellent stimulus for use in neurophysiologi- cal experiments, and especially those involving competition; they have been used extensively in previous work in the owl isthmotectal network [Asadollahi et al., 2010; Mysore et al., 2010, 2011; Mysore and Knudsen, 2011a, 2012, 2013, 2014; Asadollahi and Knudsen, 2016].

Auditory stimuli were broadband bursts generated using Tucker Davis Technologies hardware and custom MATLAB scripts. They were filtered with head-related transfer functions of a typical barn owl [Witten et al., 2008] and presented binaurally through matched earphones (ED-1914; Knowles) coupled to damping assemblies (BF-1743) inserted into the ear canals approximately 5 $\mathrm{mm}$ from the eardrums. The amplitudes of the two earphones were equalized to within $2 \mathrm{~dB}$. The binaural level of auditory stimuli at each recording site was set to $20 \mathrm{~dB}$ above its threshold.

\section{Spatial RFs}

RFs were first estimated manually by moving a stationary dot and monitoring firing responses associated with the stimulus's location. Then, a series of randomly interleaved visual stimuli were presented over a range of elevations and azimuths (typically \pm 40 $60^{\circ}$ in elevation at a sampling step of $10-12^{\circ}$ or $\pm 20-30^{\circ}$ in azimuth at a sampling step of $5-7.5^{\circ}$ from the center of the area evoking high firing rates) over 10 repetitions. Locations at which stimuli evoked responses above baseline were determined be within the site's RF (ANOVA followed by correction for multiple comparisons).

The fixed location of S1 inside the RF (Fig. 2-5) was chosen to be one of the locations that elicited responses above half-max response for azimuthal tuning curves. Across multiple presentations, the locations of $S 1$ were jittered randomly along the circumference of a circle of radius of $2^{\circ}$ centered around the nominal position of S1, to reduce the effects of habituation [Asadollahi et al., 2011; Mysore et al., 2011]. For elevational tuning curves (which were very broad in Imc) [Wang and Frost, 1991; Mahajan and Mysore, 2018], S1 was presented at one of the locations that elicited responses above half-max response, and was similarly jittered.

\section{Data Analysis}

Response firing rates to a visual stimulus presented inside the RF (either alone or with a second stimulus of either sensory modality) were calculated by computing the average firing rate during a window of 125-275 ms after stimulus onset, and then subtracting the baseline firing rate (computed from all interleaved trials) from that value. Response firing rates to an auditory stimulus presented alone were computed in a similar manner, but using a window of 5-155 ms after response onset; the earlier window was due to the short response latency to auditory stimuli.

All statistical analyses were conducted using custom MATLAB scripts. Normally distributed datasets (determined using Lilliefors tests, lillietest) utilized parametric statistical tests, otherwise nonparametric tests were used (sign-tests, signtest in MATLAB). Where applicable, the Holm-Bonferroni correction was used to correct for multiple comparisons, and significance was detected at the level of 0.05 .

To measure the effect of a distant competitor on the azimuthal tuning curve (Fig. 1), a straight line was fit to data pairs using a linear regression function in MATLAB. The intercept parameter was considered to be significantly different from zero at a significance level of 0.05 if its associated $95 \%$ confidence did not overlap with zero. Similarly, the slope parameter was considered to be dif- 
ferent from 1 at a significance level of 0.05 if its $95 \%$ confidence intervals did not overlap with 1.

The magnitude of suppression of responses to $\mathrm{S} 1$ as a function of location of S2 (Fig. 2-5) was measured by calculating the percentage change of responses to S1 and S2 over the responses to S1 alone. The locations of S2 associated with significant suppression were identified at each site by performing a one-way ANOVA (anova1 in MATLAB) on the percentage-change data followed by the standard Holm-Bonferroni correction for multiple comparisons to correct for the number of locations outside the RF at which S2 was presented (abbreviated simply as "Holm-Bonferroni correction for multiple comparisons"). For summary plots of suppression (across the population of Imc sites), all locations from each Imc site that were $\geq 15^{\circ}$ outside the RF were collected, along with the percent change in responses at those locations. These data from all sites were binned by location, and the average percentage suppression within each bin computed. Bin sizes were chosen to be large enough to minimize the number of empty bins; the bin size for elevational data (Fig. 4, 5) was larger than for azimuthal data (Fig. 2,3) because fewer locations outside the RF were available for sampling (owing to the large elevational span of Imc tuning curves; Fig. 4) [Wang and Frost, 1991; Mahajan and Mysore, 2018], resulting in a sparser dataset. The locations of S2 associated with significant suppression in this summary data were also identified by performing a one-way ANOVA (anoval in MATLAB) on the percentage-change data within each bin followed by the Holm-Bonferroni correction for multiple comparisons (to correct for the number of bins, i.e., locations).

\section{Results}

\section{Effect of a Competitor on Imc Spatial Tuning Curves}

To examine if Imc neurons themselves expressed signatures of stimulus competition, we asked whether multiunit Imc responses to a stimulus inside the RF were modulated by the presence of a competing stimulus outside the RF. To this end, we adopted a protocol used previously to investigate stimulus competition in the OTid (Fig. 1a, b) [Mysore et al., 2010].

We measured azimuthal tuning curves of Imc neurons by varying the azimuthal location of a looming visual stimulus (S1; loom speed $=6 \%$; Fig. 1a). Looming stimuli have been shown to be very effective in driving the responses of neurons in the midbrain selection network, with faster loom speeds evoking greater neural responses, and thereby corresponding to stronger stimuli [Asadollahi et al., 2010; Mysore et al., 2010; Mahajan and Mysore, 2018]. Here, we compared these responses to $S 1$ alone to those when a second stimulus (S2) was simultaneously presented far outside the RF (Fig. 1b). S2 was also a looming visual stimulus, typically looming $4^{\circ} / \mathrm{s}$ faster than $\mathrm{S} 1$ $\left(10^{\circ} ; n=17\right.$ sites $)$, and was presented $\geq 30^{\circ}$ away from the $\mathrm{RF}$ center, at the same elevation as $\mathrm{S} 1$. The single stimulus and two-stimulus conditions were randomly interleaved.
We found that when S2 was present, responses to stimuli located within the RF decreased (Fig. 1c). To quantify the nature and strength of suppression, we compared the responses to both stimuli against those to $\mathrm{S} 1$ alone as a scatter plot, and fit a straight line to the response pairs obtained from locations identified as being within the RF of the Imc recording site (Fig. 1d, filled circles). We found that the straight line had a slope of $0.65(p<0.05$ with respect to a slope of 1$)$, and a $y$-intercept of 4 ( $p>0.05$ with respect to an intercept of 0 ), demonstrating that S2 suppressed the responses of this multiunit site in a divisive, but not subtractive, manner. Across $n=17$ sites, we found that $12(70 \%)$ exhibited a significant suppression. Across these sites, the median slope of the best-fit line was 0.65 , and was significantly less than $1(p<0.05)$, whereas the median $y$-intercept was not different from zero (median $=0.33 ; p>0.05$; Fig. 1e).

These data demonstrated that Imc responses reflect competitive interactions. The typical influence of a stronger competitive stimulus than $\mathrm{S} 1$ ( $4 \%$ faster), located outside of the RF, was powerful divisive inhibition $(40 \%$ suppression on average; Fig. 1e). This direct demonstration of the encoding of stimulus competition by Imc neurons motivated us to investigate the spatial dependence of competitive interactions in the Imc.

\section{Effect of Visual S2 Placed at Different Locations along the Azimuth}

To investigate how the location of S2 affects the responses of Imc neurons to S1, we systematically varied the azimuth of S2, while maintaining the location of S1 fixed at the RF center (Fig. 2a) [Mysore et al., 2010]. Both S1 and S2 were looming visual stimuli as before, with S2 being $4^{\circ}$ s faster than S1, and therefore evoking stronger responses as expected. S2 was placed at several locations outside the RF. These included locations that were encoded by the same side of the brain as S1 (denoted by green data and azimuthal values $\geq-15^{\circ}$ ), as well as locations encoded by the opposite site of the brain (denoted by purple data and azimuthal values $<-15^{\circ}$ ) [Knudsen, 1982]. In addition, S2 was also placed at locations inside the RF, but not overlapping with S1 (denoted by blue data). The locations of S2 when presented with S1 were interleaved randomly with the presentation of S1 alone and S2 alone.

We found that when S2 was outside the RF, Imc responses to S1 were suppressed strongly at many locations outside the RF (Fig. 2b; raster; $p<0.05$, one-way ANOVA followed by Holm-Bonferroni correction for multiple comparisons to correct for the number of locations outside the RF). 


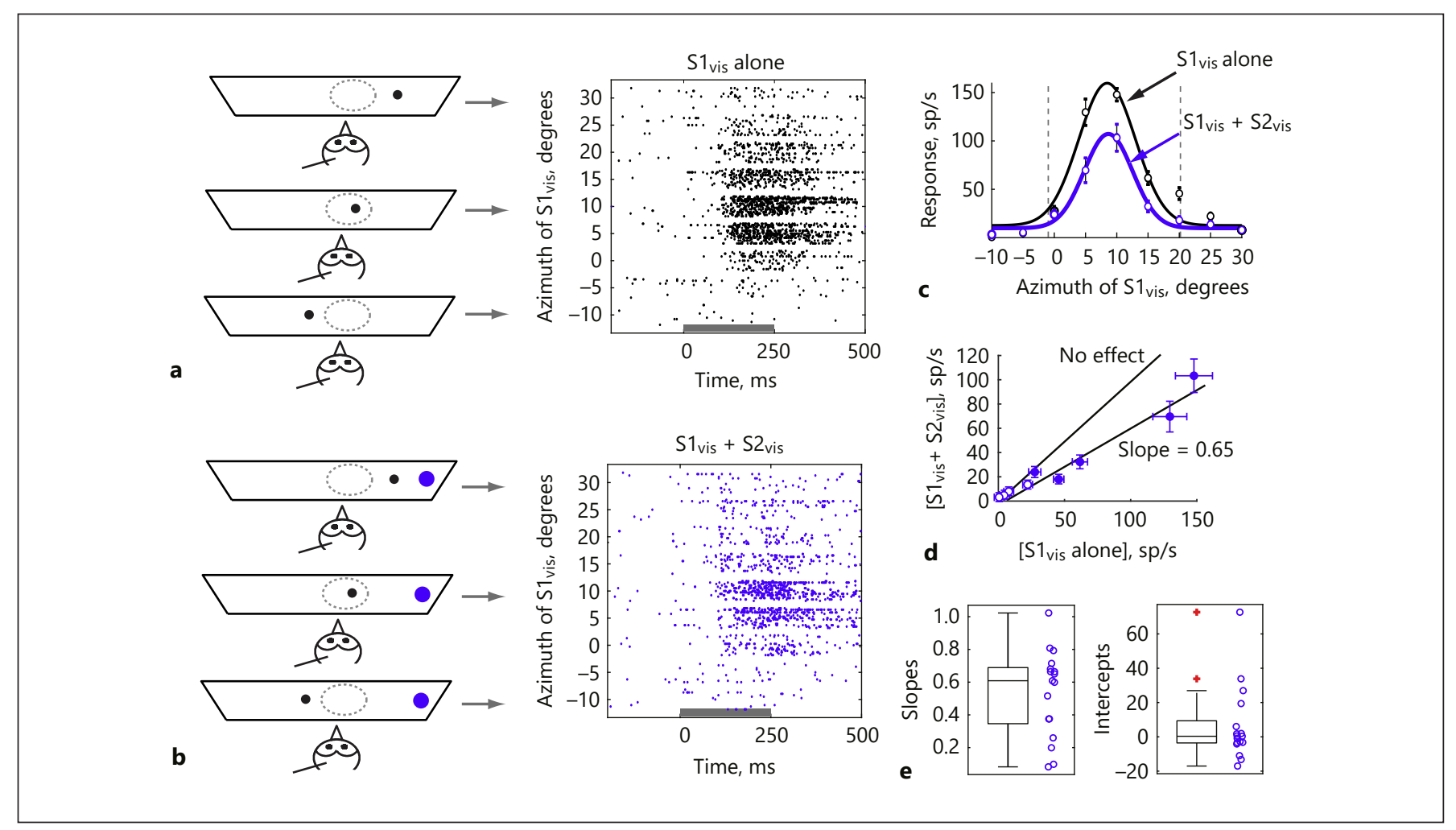

Fig. 1. Effect of visual competitor (S2) on visual tuning curves in the Imc. a Left: schematic showing the stimulus protocol for measuring the visual azimuthal tuning curve. Depicted is an owl (with an electrode in the Imc) looking at a tangent monitor. Black dot, S1, looming visual dot; dotted circle, RF of the Imc site. Panels correspond to locations in the raster plot (right), indicated by arrows. Right: raster plot of neural responses to S1 alone from an example Imc site. Dark grey bar, duration of stimulus presentation (250 $\mathrm{ms}$ ); loom speed of S1 $=6 \%$ s. b Left: schematic showing stimulus protocol for measuring visual azimuthal tuning curve in the presence of a distant competitor $\left(>30^{\circ}\right.$ away from S1). Conventions are as in a. Blue dot, S2, also a looming visual stimulus; larger dot size denotes faster loom. Right: raster plot of neural responses to S1 and S2 presented together at the same site; paired presentation interleaved randomly with S1 alone. Loom speed of S2 $=10^{\circ} /$ s. c Tuning curves obtained by plotting response firing rates at the different

To quantify the suppression as a function of S2 locations outside the RF, we computed the percentage change in the response to S1 and S2 presented together with respect to the responses to $\mathrm{S} 1$ alone (Fig. 2e). We found that $\mathrm{S} 2$ exerted suppression on responses to $\mathrm{S} 1$ from locations just outside the RF (approx. $15^{\circ}$ from the RF center) and far distant from the RF center (approx. $30^{\circ}$ from the RF center; Fig. 2e; data points outside the dotted vertical lines). Importantly, suppression was found both when S2 was in the same hemifield as S1 and when it was in the opposite hemifield (Fig. 2e). locations of S1; firing rates were obtained by counting spikes in the rasters within a fixed window following stimulus onset. Curves represent best-fit Gaussians to responses. Error bars indicate the mean \pm SEM. Dashed vertical lines, extent of RF. d Scatter plot of responses to the paired presentation against responses to $S 1$ alone; same data as in C, replotted. Filled dots, data for locations within the RF; open dots, data for locations outside the RF; gray line, line with slope of unity (representing no change); blue line, best-fit line to the filled dots; slope $=0.65$ (significantly different from 1 , indicating response suppression) and $y$-intercept of -4.0 spikes/s (not significantly different from 0 ). e Summary of slopes and intercepts of best-fits across the population of $n=17$ Imc sites. Left: boxplot and distribution of slopes; each circle represents one site; median $=0.61(p<0.05$; rank-sum test against 1$)$. Right: boxplot and distribution of $y$-intercepts; median $y$-intercept value $=0.33(p>$ 0.05 ; rank-sum test against 0 ).

We repeated this experiment across $n=44 \mathrm{Imc}$ sites and found that 41 sites exhibited response suppression for at least one location of S2 (93\%). To visualize the data, we pooled the changes in firing rate based on the (binned) spatial location of $\mathrm{S} 2$ when it was outside the RF (Fig. 2f). Across the population, S2 significantly suppressed S1 responses from all locations outside the RF tested (Fig. 2f; $p<0.05$, one-way ANOVA followed by Holm-Bonferroni correction for multiple comparisons), both when it was ipsilateral to S1 as well as contralateral. At S2 locations $>-15^{\circ}$ in azimuth, i.e., locations encoded by the same side 


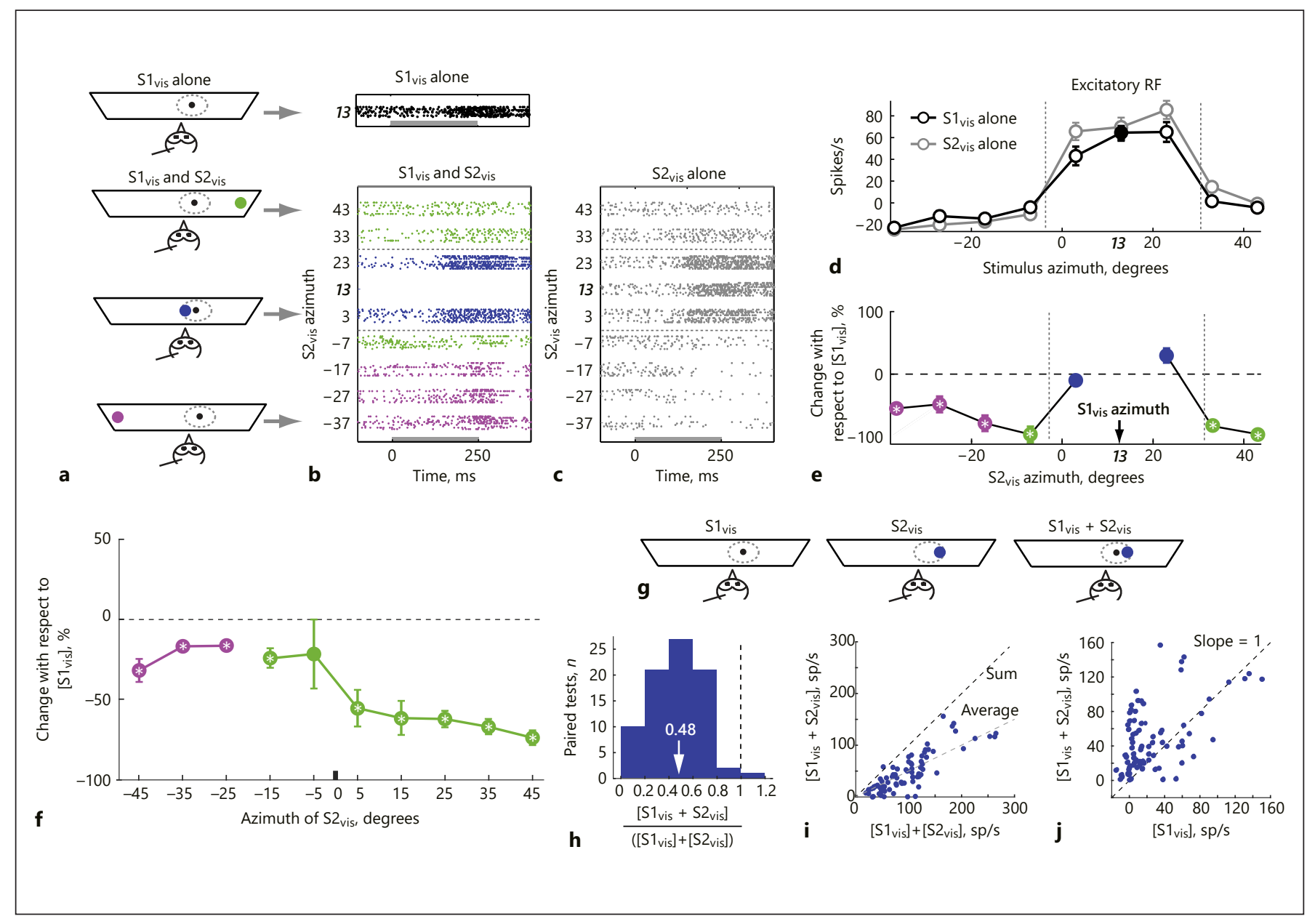

Fig. 2. Effect of the azimuthal location of a visual competitor (S2) on Imc responses to a visual stimulus (S1) inside the RF. a Schematic showing the stimulus protocol. Colors correspond to qualitatively different positions of S2; details are given in $\mathbf{b}$. $\mathbf{b}$ Raster plot of responses to $S 1$ alone (top panel), and to the paired presentation of S1 and S2 (bottom panel) from an example Imc site; S1 location was fixed at $+13^{\circ}$. Positive azimuthal values represent contralateral locations (to the recording site), and negative values to ipsilateral locations. Horizontal dotted lines indicate the extent of RF mapped with stimulus S2. Blue data correspond to locations of S2 inside the RF; green data to azimuthal locations encoded by the same hemisphere of the OT that encodes S1 (i.e., azimuths $>-15^{\circ}$ ), and purple data to locations encoded by the opposite hemisphere of the OT that encodes S1. Responses to S1 when S2 was presented at the same location (overlapping with $\mathrm{S} 1$ ) are whited out. Loom speeds, $\mathrm{S} 1=6 \% \mathrm{~s} ; \mathrm{S} 2=12 \%$ s. c Raster plot of responses to S2 alone. Stimulus presentations of different kinds (S1 alone, S2 alone, etc.) and at different locations were interleaved randomly. d Azimuthal spatial tuning curves of this example site measured independently with S1 or S2. Vertical dotted lines, extent of RF. e Percent change in responses to paired presentation of $S 1$ and $S 2$ compared to responses of S1 alone, as a function of location of S2 at the example site. Dotted horizontal line, $0 \%$ change. Colors are the same as in b (bottom panel). Error bars denote the mean \pm SEM; the size of dot representing the mean is often larger than the extent of SEM.
* Suppression is significantly different from 0; one-way ANOVA followed by Holm-Bonferroni correction for multiple comparisons; tested only for locations outside of the RF. $f$ Population summary plot of percent change in responses to paired presentation compared to responses to S1 alone, as a function of location of S2, across $n=41 \mathrm{Imc}$ sites. Average loom speed of S1 $=5.9^{\circ} \mathrm{s} ; n=44$ sites; loom speed of S2 was higher than S1 by $4.2 \%$, on average. g-j Population summary of responses when S2 was also inside the RF. "[S]" denotes firing rate responses to stimulus condition S. $\mathbf{g}$ Schematic of the different stimulus conditions. $\mathbf{h}$ Histogram of the integration ratio (ratio of responses to paired presentation over the sum of individual responses) from $n=92$ paired presentations across 44 Imc sites. White arrow, median; dashed line = integration ratio of 1 . i Scatter plot of data in $\mathbf{h}$ showing spread of responses to paired presentation as a function of the sum of individual responses. The dashed black line is the line with a slope of 1 , denoting responses to paired presentation that equal the sum of individual responses to S1 and S2 (i.e., reflecting an "additive" integration rule). The dashed grey line has a slope of 0.5 , denoting responses to paired presentation that equal the average of the individual responses (i.e., reflecting an "averaging" integration rule). $\mathbf{j}$ Scatter plot of data in $\mathbf{h}$ showing the spread of responses to paired presentation as a function of the responses to $\mathrm{S} 1$. The dashed black line is the line with a slope of 1, denoting responses to paired presentation that are the same as responses to just S1. 
of the brain as S1 [Knudsen, 1982], S2 exhibited powerful suppression, ranging from $-24.62 \%$ to $-79.33 \%$. Notably, for S2 locations encoded by the opposite side of the brain $\left(<-15^{\circ}\right.$ azimuth), the suppression was weaker, ranging from $-19.25 \%$ to $-35.37 \%$. Although weaker $(p<0.05$; $t$ test of pooled suppression values when S2 was on the same side vs. on the opposite side), the existence of significant suppression when S2 occupied these far contralateral locations to $\mathrm{S} 1$ was notable. These data demonstrated that positions of a competitor across all tested locations along the azimuth, even represented by the opposite side of the brain, effectively inhibited Imc responses, indicating that stimulus competition in the Imc was global along the azimuthal axis.

Next, we examined the effect of S2 on responses to S1, when S2 was also inside the RF, i.e., stimulus integration. We found that the effects were markedly different. Responses to the paired presentation were greater than the responses to S1 alone, consistent with both stimuli being able to excite the Imc site (Fig. 2e; data points within the dotted vertical lines). To quantify the nature of the modulation across sites, we computed an integration ratio at every location of S2 tested inside the RF. This was defined as the ratio of the responses to paired presentation of $S 1$ and $S 2$ over the sum of the individual responses to $S 1$ and $\mathrm{S} 2$, i.e., $\left[\mathrm{S} 1_{\mathrm{vis}}+\mathrm{S} 2_{\mathrm{vis}}\right] /\left(\left[\mathrm{S} 1_{\mathrm{vis}}\right]+\left[\mathrm{S} 2_{\mathrm{vis}}\right]\right)$. We plotted the distribution of this ratio (Fig. $2 \mathrm{~h} ; n=92$ paired presentations from 44 Imc sites).

The ratios were primarily less than 1 (in all but 1 case), indicating that the response to the paired presentation was less than the sum of the individual responses, i.e., sub-additive. The median ratio was 0.48 , i.e., the response to the paired presentation was typically 0.48 times the summed response, indicating that the typical effect of paired stimulus integration was one of averaging their individual responses. To examine whether the nature of integration (sub-averaging vs. super-averaging, i.e., ratio $<0.5$ vs. ratio $>0.5$ ) depended on the strength of the responses to individual stimuli, we also plotted the responses to the paired presentation against the sum of the responses to the individual presentations (Fig. 2i). When the responses to S1 and S2 were both weak, responses to the paired presentation varied over the full range. However, when the individual responses were strong, responses to the paired presentation were typically super-averaged. Responses to the paired presentation were greater than responses to $S 1$ alone in the majority of the cases (Fig. 2j). This was consistent with the fact that $\mathrm{S} 2$ typically evoked stronger responses than $\mathrm{S} 1$ (as expected because of the faster looming speed of S2), and with the observed response averaging.

Stimulus Competition in the Owl Imc

\section{Effect of Auditory S2 Placed at Different Locations} along the Azimuth

To evaluate whether the effects of a distant competitor were similar across sensory modalities, we performed these same tests utilizing a visual S1 (as before), but with an auditory S2, at a subset of the Imc sites. This allowed an assessment of cross-modal competition as well as cross-modal integration in the Imc. The auditory S2 was a broadband noise burst, with a binaural level of $20 \mathrm{~dB}$ above the threshold of the site. The auditory S2 was presented at various locations outside and inside the RF along the azimuth (similar to the visual S2, above). The presentations of S1 alone, S2 alone, and S1 paired with S2 (at different S2 locations) were all interleaved randomly.

First, we examined how the auditory S2 affected responses to S1 when S2 was presented outside the RF at different azimuthal locations (Fig. 3a). We found that Imc responses to a visual $\mathrm{S} 1$ were suppressed by an auditory S2 at all locations outside the RF (Fig. 3b). The percentage change in the response to S1 and S2 presented together with respect to the responses to $\mathrm{S} 1$ alone confirmed that an auditory S2 exerted suppression on responses to S1 from locations just outside the RF (approx. $10^{\circ}$ from the $\mathrm{RF}$ center) and far distant from the RF center (approx. $30^{\circ}$ from the RF center; Fig. 3e; data points outside the dotted vertical lines; $p<0.05$, one-way ANOVA followed by Holm-Bonferroni correction for multiple comparisons). Importantly, suppression was found both when an auditory S2 in the same hemifield as S1 (Fig. 3e, green), and when it was in the opposite hemifield (Fig. 3e, purple).

Among the $n=17$ sites tested, the auditory S2 produced significant suppression from at least one location in 14 sites (82\%; at each site: $p<0.05$, one-way ANOVA followed by Holm-Bonferroni correction for multiple comparisons). For these sites, we quantified S2's effect as a percentage change in responses to $\mathrm{S} 1$ alone, and plotted it as a function of binned S2 locations. Across the sites, we found that $\mathrm{S} 2$ suppressed $\mathrm{S} 1$ responses significantly across space (Fig. 3f; $p<0.05$, one-way ANOVA followed by Holm-Bonferroni correction for multiple comparisons). At S2 locations encoded by the same side of the brain as S1, S2 exhibited strong suppression, ranging from $-46.88 \%$ to $-81.08 \%$. At S2 locations represented by the opposite site of the brain, the suppression was significant, ranging from $-33.79 \%$ to $-49.85 \%$, but weaker than on the same side $(p<0.05 ; t$ test of pooled suppression values when S2 was on the same side vs. on the opposite side, one-way ANOVA followed by Holm-Bonferroni correction for multiple comparisons). Thus, an auditory competitor exerted similar, albeit slightly weaker (overall), 


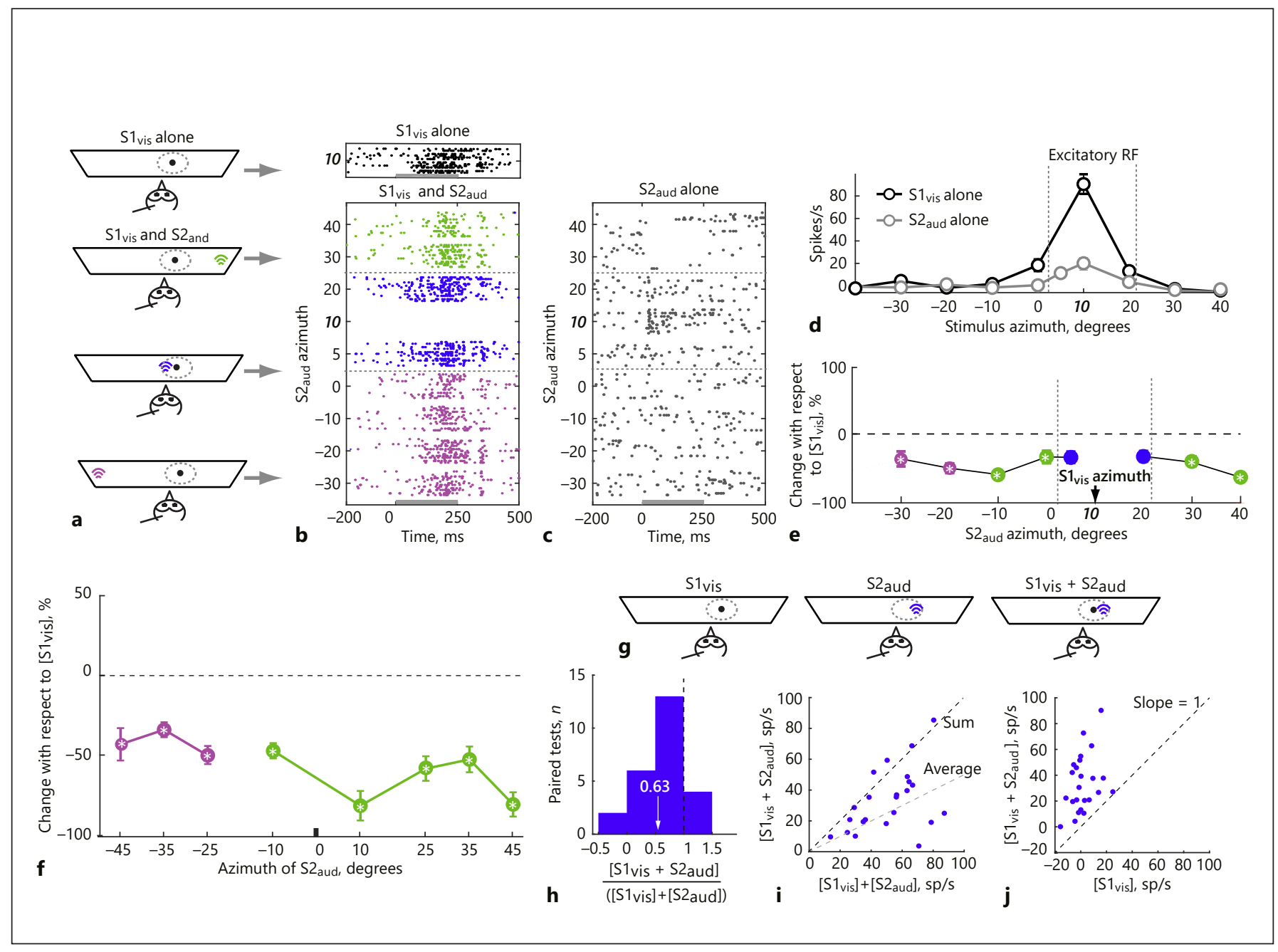

Fig. 3. Effect of the azimuthal location of an auditory competitor (S2) on Imc responses to a visual stimulus (S1) inside the RF. Panels are organized in an identical manner and all conventions are the same as in Figure 2. a Schematic of the experiment. b-f Effect of location of S2 on Imc responses to S1, when S2 was outside the RF. b-e Responses from an example Imc site. S1 location was fixed

suppressive effects from far-ranging locations along the azimuthal plane, much like a visual competitor.

Next, we examined how the auditory S2 affected responses to $\mathrm{S} 1$ when $\mathrm{S} 2$ was also presented inside the RF (Fig. $3 \mathrm{~g}$ ). The ratio of the paired responses to the sum of the individual responses was less than 1 (sub-additive) in nearly all cases, with a median of 0.63 , indicating response averaging (Fig. $3 \mathrm{~h} ; p>0.05$, signed-rank test against 0.5 ; $n=27$ paired presentations across 17 sites). When the responses to S1 and S2 were both weak, responses to the paired presentation ranged from sub-averaging to superaveraging. However, when the individual responses were at a $10^{\circ}$ azimuth. Loom speed of $\mathrm{S} 1=6^{\circ} / \mathrm{s}$; the binaural level of S2 was $20 \mathrm{~dB}$ above the threshold. f Summary of data from $n=14 \mathrm{Imc}$ sites. $\mathbf{g}$-j Effect of S2 on Imc responses to S1, when S2 was also inside the RF; data from $n=27$ paired presentations across 17 Imc sites. Average loom speed of $\mathrm{S} 1=5.4^{\circ} / \mathrm{s}$; the average binaural level of $\mathrm{S} 2$ was $20 \mathrm{~dB}$ above the threshold for each site.

strong, responses to the paired presentation were predominantly super-averaged. Responses to the paired presentation were greater than responses to $\mathrm{S} 1$ alone in the majority of the cases (Fig. 3j), consistent with the observed averaging.

\section{Effect of Visual S2 Placed at Different Locations along the Elevation}

It is well established that whereas Imc neurons encode visual space with asymmetric RF [Wang and Frost, 1991; Mahajan and Mysore, 2018], Imc visual RFs are elongated along the elevation (tall along elevation and narrow along 


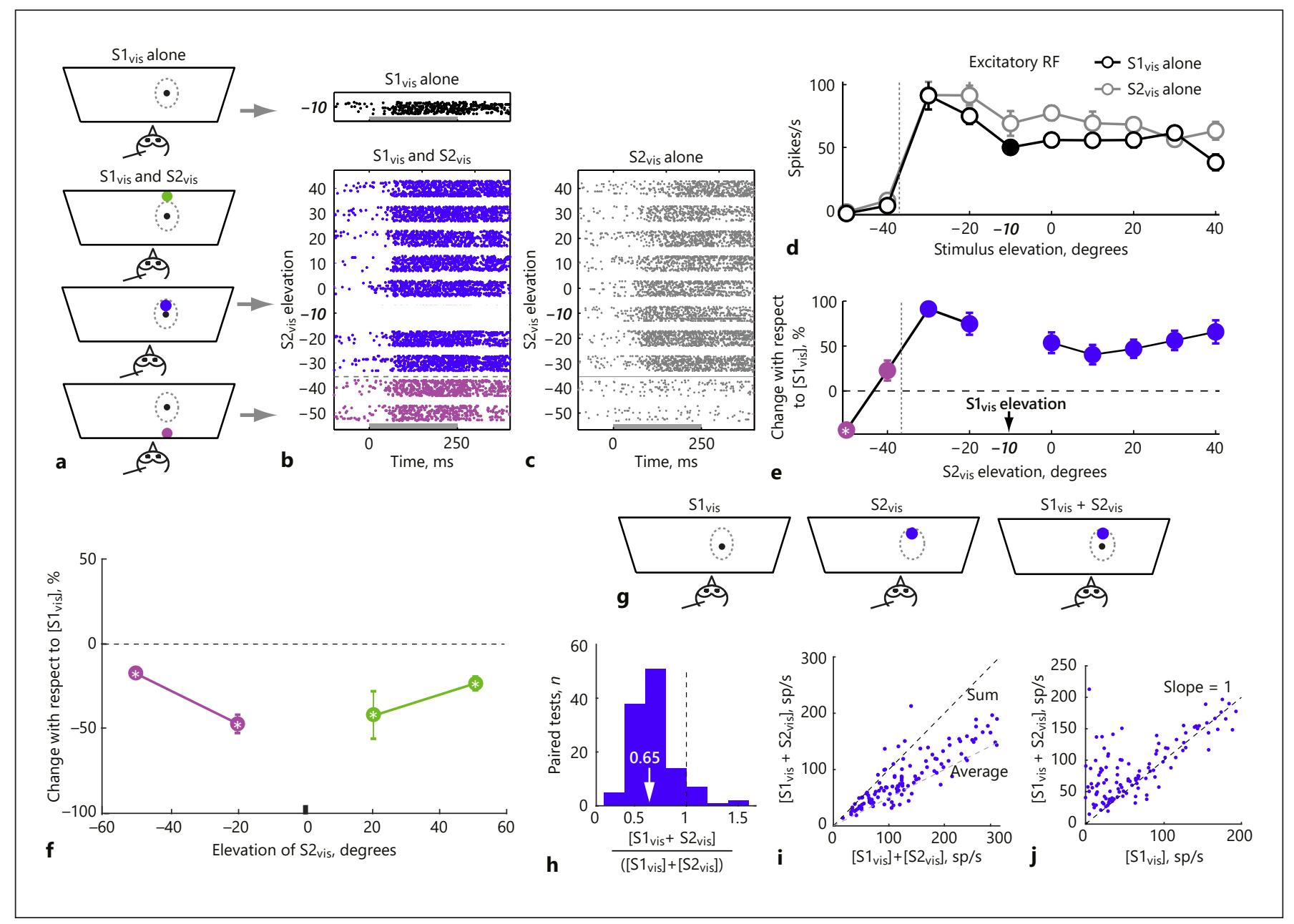

Fig. 4. Effect of the elevational location of a visual competitor (S2) on Imc responses to a visual stimulus (S1) inside the RF. Panels are organized in an identical manner and all conventions are the same as in Figure 2. a Schematic of the experiment. b-f Effect of location of S2 on Imc responses to S1, when S2 was outside the RF. b-e Responses from an example Imc site. S1 location was fixed at $-10^{\circ}$ elevation. Loom speeds, S1 $=4 \%$ s; S2 $=10 \%$ s. The span of eleva-

azimuth), and, in addition, frequently have multi-peaked tuning curves [Mahajan and Mysore, 2018]. Therefore, we were interested in investigating stimulus interactions in the Imc along the elevation, and comparing the results to those along the azimuth.

To this end, we recorded the responses of Imc neurons to a looming visual stimulus (S1) inside the RF, either by itself, or while simultaneously presenting a second, competing visual stimulus (S2) at various locations both inside and outside the RF along the elevation (Fig. 4a). Consistent with the published reports [Wang and Frost, 1991; Mahajan and Mysore, 2018], we found that Imc tional RF of this site is large, extending from $-35^{\circ}$ to at least $+40^{\circ}$ (consistent with published literature [Wang and Frost, 1991; Mahajan and Mysore, 2018]).f Summary of data from $n=15$ Imc sites. g-j Effect of S2 on Imc responses to S1, when S2 was also inside the RF; data from $n=128$ paired presentations across 26 Imc sites. Average loom speed of S1,5.3\% $\mathrm{s}$; loom speed of S2 was higher than S1 by $4^{\circ} / \mathrm{s}$, on average.

tuning curves were very broad along elevation (Fig. 4d; average span of tuning curves $=73^{\circ} ; n=26$ sites). Because of this large extent of Imc elevational tuning curves, there were typically far fewer locations outside the RF to sample (than in the case of our experiments along the azimuth; Fig. 2, 3). For instance, in the example Imc site shown (Fig. 4), the elevational RF ranged from $-35^{\circ}$ to at least $+40^{\circ}$, leaving just two S2 locations outside the RF (Fig. 4d, e).

At the example Imc site, we found that when S2 was located at $-50^{\circ}$ elevation, a location well outside the elevational RF, it effectively suppressed the responses to S1 
(Fig. 4e). However, for the location just outside the RF ( $-40^{\circ}$ elevation; Fig. $\left.4 \mathrm{e}\right), \mathrm{S} 2$ did not suppress responses to S1. This was explained, potentially, by the fact that paired responses when $\mathrm{S} 2$ elevation was $-40^{\circ}$ represented a transition from response enhancement when S2 was inside the RF ( $\mathrm{S} 2$ elevation $=-30^{\circ}$ ) to response suppression when S2 was outside the RF (S2 elevation $=-50^{\circ}$ ).

We repeated this experiment across $n=26$ Imc sites and found that of the ones at which we were able to place S2 outside the RF (24 sites), 15 sites (62.5\%) exhibited a significant suppression for at least one S2 location (at each site: $p<0.05$, one-way ANOVA followed by HolmBonferroni correction for multiple comparisons). We visualized the data from these 15 sites by pooling the changes in firing rate as a function of the (binned) spatial location of S2 when it was outside the RF (both just outside and far outside). Because there were fewer outside RF locations along the elevation, the number of samples was fewer, resulting in the need for a wider bin size than along the azimuth (Fig. 3f vs. Fig. 4f).

We found that on average, the range of suppression due to S2 located outside the RF varied from $-17.56 \%$ to $-47.4 \%$ for S2 locations both below the horizon (lower elevations; Fig. 4f, purple data) and above the horizon (upper elevations; Fig. 4f, green data). Thus, a wide range of outside-RF positions of a competitor stimulus along the elevation effectively inhibited Imc responses, just as along the azimuth.

Next, we investigated stimulus integration along the elevational axis by examining how a visual S2 affected responses to S1, when S2 was also presented inside the RF (Fig. 4e; data points at elevations greater than $-35^{\circ}$ ). As with azimuthal integration, we found that paired responses were enhanced over responses to $\mathrm{S} 1$ alone (Fig. 4j).

To quantify this effect across the population, we computed the integration ratio from $n=128$ paired presentations obtained from $26 \mathrm{Imc}$ sites (Fig. 4h). These ratios were predominantly sub-additive $(<1)$, and superadditive $(>1)$ only in a minority of the cases (9/128 data pairs). The median integration ratio was 0.65 , indicating a typical effect that was slightly stronger than averaging $(p<0.05$; signed-rank test), but still sub-additive. This effect occurred both when the individual responses were weak as well as when they were strong (Fig. 4i), and appeared as a consistent enhancement with regard to responses to S1 alone (Fig. 4j).

These results indicated that the integration of responses by Imc neurons to paired, within-RF stimulus presentations along the elevation was qualitatively similar to that along the azimuth (Fig. 2, 3).

\section{Effect of Auditory S2 Placed at Different Locations} along the Elevation

Finally, we examined the nature of stimulus interactions when S2 was an auditory stimulus. To this end, we presented an auditory $\mathrm{S} 2$ (broadband stimulus; $\mathrm{ABL}=20$ $\mathrm{dB}$ above site threshold), at different locations along the elevational axis (Fig. 5a, c), with a looming visual S1 presented inside the RF. At the example site, we found that when an auditory S2 was outside the RF at lower elevations, S2 effectively suppressed the responses to S1 (Fig. 5e).

Across our population of 9 sites, we found that $\mathrm{S} 2$ produced significant suppression of responses to $S 1$ from at least one location in 8 sites $(p<0.05$, one-way ANOVA followed by Holm-Bonferroni correction for multiple comparisons). The magnitude of suppression due to auditory S2 was $-33.42 \%$ on average for locations above the horizon, and $-54.13 \%$ on average for locations below the horizon (Fig. 5f).

When $\mathrm{S} 2$ was presented inside the RF, the integration ratio was predominantly sub-additive (Fig. $5 \mathrm{~h} ;<1$ in all but 3 cases), with a median value of 0.59 , indicating a typical effect of averaging. This effect occurred both when the individual responses to both $\mathrm{S} 1$ and $\mathrm{S} 2$ were weak, and when they were strong (Fig. 5i), and reflected an overall enhancement in the paired responses over responses to $\mathrm{S} 1$ alone (Fig. 5j). Thus, the effect of audio-visual integration along the elevational axis was sub-additive, and close to response averaging (as in the visual-visual case).

Taken together, the above results revealed that, despite the asymmetry in the nature of the elevational versus azimuthal encoding of space in the Imc [Wang and Frost, 1991; Mahajan and Mysore, 2018], there were no systematic differences between stimulus interactions along the elevational versus azimuthal axes.

\section{Discussion}

The results of this study revealed functional properties of an inhibitory nucleus, Imc, known to play a vital role in stimulus selection in the midbrain selection network [Wang et al., 2004; Knudsen, 2011; Mysore and Knudsen, 2013]. Whereas previous work has investigated in detail the properties of the RF of Imc neurons by presenting single stimuli to the animal [Wang and Frost, 1991; Mahajan and Mysore, 2018], this study investigated Imc response properties to competing stimuli presented simultaneously. Results revealed that Imc neurons exhibit global signatures of stimulus competition, expressed as 


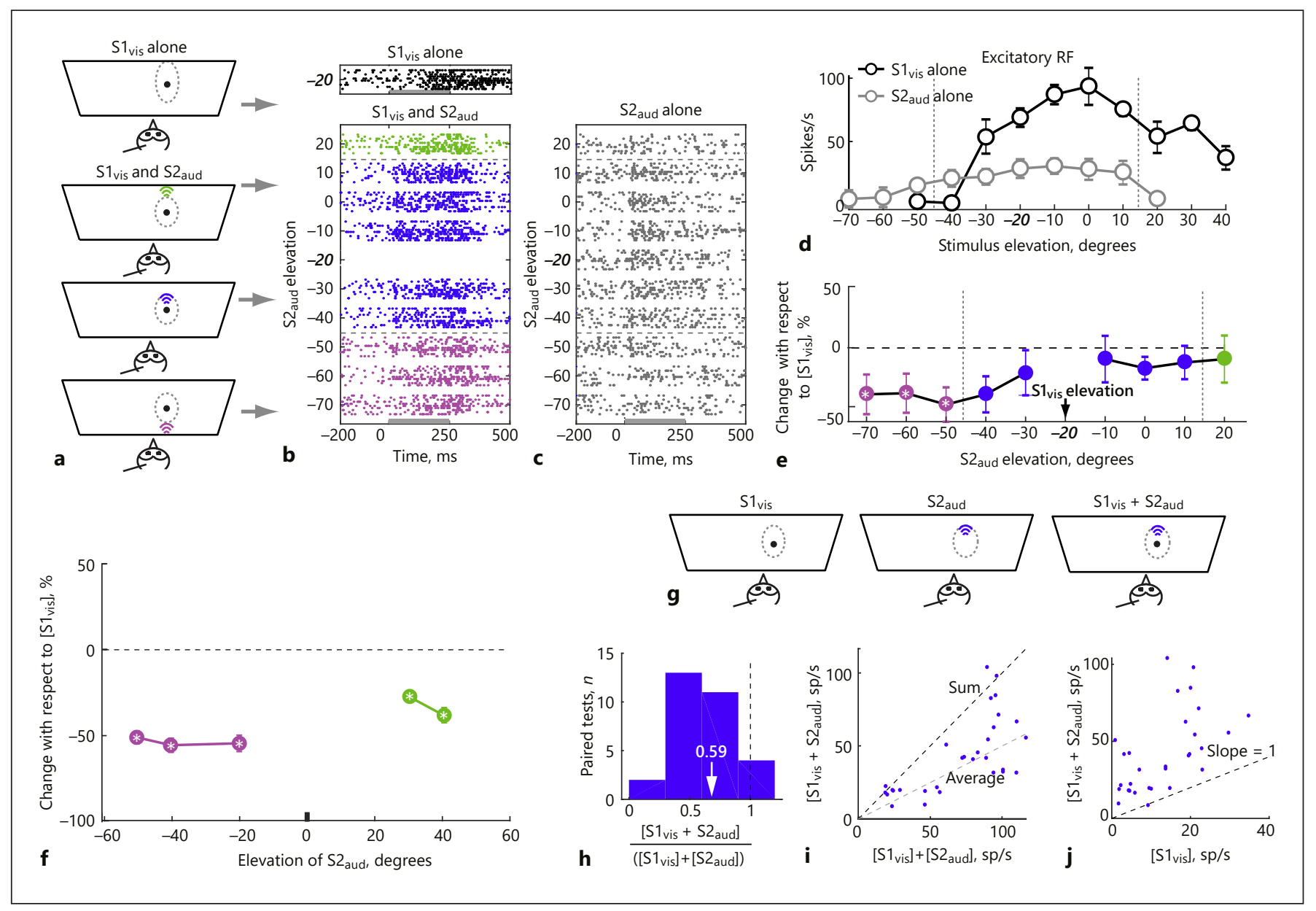

Fig. 5. Effect of the elevational location of an auditory competitor (S2) on Imc responses to a visual stimulus (S1) inside the RF. Panels organized in an identical manner to Figure 2; all conventions are the same as in Figure 2. a Schematic of the experiment. b-f Effect of location of S2 on Imc responses to S1, when S2 was outside the RF. b-e Responses from an example Imc site. S1 location was

the suppression of responses to stimuli inside the RF by stimuli outside. The nature of the suppression was divisive, similar to that reported in the OT [Mysore et al., 2010].

These results refine our current understanding of the role of the Imc in spatial selection. They suggest that beyond its role in supplying neural inhibition that is necessary for competitive interactions in the OTid, the Imc may itself be an active site of stimulus competition. The patterns of Imc activity reported here are consistent with it maintaining a map of relative priority, a key task of the midbrain attention network. To the best of our knowledge, this is the first evidence supporting the encoding of relative priority maps by inhibitory neurons. fixed at $-20^{\circ}$ azimuth. Loom speed of S1, $6 \%$ s; the binaural level of S2 was $20 \mathrm{~dB}$ above the threshold. $\mathbf{f}$ Summary of data from $n=8$ Imc sites. $\mathbf{g}$-j Effect of S2 on Imc responses to S1, when S2 was also inside the RF; data from $n=33$ paired presentations across 8 Imc sites. Average loom speed of S1,4.7\% of S2 was $20 \mathrm{~dB}$ above the threshold for each site.

\section{Suppression by Stimuli Outside the RF}

In this study, we systematically examined the spatial dependence of stimulus interactions in the Imc. Specifically, competitors located far outside the RF were very effective in suppressing the responses to a visual stimulus presented inside the RF. This suppression was global in its effect - competitors located anywhere along the elevation or azimuthal axis exerted response suppression. Notably, competitors that were encoded by the same side of the midbrain as the RF stimulus, as well as those that were encoded by the opposite side of the brain, were both effective at exerting suppression. The suppression due to contralateral competitors was, however, weaker than that due to ipsilateral competitors, similar to the effect 
reported in other key structures in the midbrain selection network [Asadollahi et al., 2010; Mysore et al., 2010]. These suggest that cross-hemispherical suppressive influences are weaker that intra-hemispherical suppression.

Furthermore, global competitive suppression was independent of the sensory modality of the competitor. Both visual and auditory competitors were effective in driving competitive suppression of Imc responses, with the visual competitors being more effective in certain cases (Fig. 4b vs. Fig. 3b). These results are consistent with similar findings in the other midbrain structures [Asadollahi et al., 2010; Mysore et al., 2010], and are likely due to the differing efficacies of a looming visual stimulus versus noise bursts at driving responses in the midbrain network (looming dots typically evoke stronger responses) [Mysore et al., 2010, 2011].

\section{Integration of Stimuli within the RF}

In contrast to the suppressive effects of a distant competitor on Imc responses, a second stimulus presented within the RF resulted in a sub-additive integration of the individual responses. The typical effect was averaging of the individual responses for all S2 cases (visual or auditory, and S2 placed at different elevations or azimuths inside the RF) except along visual elevation, where it was slightly stronger than response averaging. Thus, there were no systematic differences in the rules of integration between azimuth and elevation or between sensory modalities. The former was notable considering the wellknown asymmetry in the encoding of elevational versus azimuthal space by Imc neurons [Wang and Frost, 1991; Mahajan and Mysore, 2018]. Response averaging by Imc neurons of stimuli inside the RF is consistent not only with similar observations in the SCid/OTid [Li and Basso, 2005; Alvarado et al., 2007; Mysore et al., 2010], but also with reports of response averaging in the visual cortex [Reynolds et al., 1999].

\section{Classical versus Competitive Surround}

The properties of integration reported here are consistent with Imc neurons possessing classical inhibitory surrounds. By contrast, the suppression of responses to a stimulus inside the RF by a discrete stimulus outside the $\mathrm{RF}$ is not consistent with the notion of the classical inhibitory surround, but rather, reflects the presence of a competitive surround.

In this context, it is important to note that classical surrounds cannot automatically predict the existence (or properties of) competitive surrounds, and vice-versa
[Mysore et al., 2010]. This is because classical surrounds, which are typically measured by presenting a single stimulus of increasing sizes [Sceniak et al., 1999; Mysore et al., 2010], have fundamentally different properties from those of the global surrounds, which are measured by presenting two discrete stimuli [Rizzolatti et al., 1974; Mysore et al., 2010; Falkner et al., 2010]. First, it is well established that the strength of the classical inhibitory surrounds drops off from the center of the RF [Sceniak et al., 1999; Mysore et al., 2010]. By contrast, the strength of the competitive surround is nearly constant, and at times greater at larger distances from the excitatory center [Rizzolatti et al., 1974; Falkner et al., 2010; Mysore et al., 2010]. As a result, the spatial extents of the two surrounds are markedly different: classical surrounds are typically local, and extend to a limited extent beyond the excitatory center, whereas competitive surrounds extend typically globally [Rizzolatti et al., 1974; Falkner et al., 2010; Mysore et al., 2010]. Second, published results indicate that neurons that mediate global competitive surrounds of the kind reported here must be mutually coupled [Mysore et al., 2010], whereas no such requirement exists for the neurons that subserve local, classical inhibitory surrounds. Third, classical and competitive inhibitory surrounds serve different functions. Classical surrounds shape the responses of neurons to single stimuli inside their RF, whereas competitive surrounds, which are activated by a second stimulus outside the RF, sub-serve stimulus competition. For these reasons, not only are the two forms of surround conceptually distinct, they must necessarily be implemented by two different circuit mechanisms. Indeed, focally silencing Imc neurons has been shown to abolish competitive suppression in the OTid, but does not affect OTid RFs, nor responses to single stimuli inside the RF [Mysore and Knudsen, 2013]. Potential mechanisms that might underlie these surrounds in the Imc are discussed next.

\section{Sources of Inhibition Underlying Global Competitive} Surround in the Imc

The Imc is the source of global inhibition observed in the OTid [Mysore et al., 2010; Mysore and Knudsen, 2013]. What then, is the source of global inhibition that is necessary to support the response suppression observed in the Imc?

A plausible source is intra-Imc inhibition, the need for which has been investigated in computational modeling work [Mysore and Knudsen, 2012]. The existence of long-range inhibition within the Imc is supported by anatomical and immunohistochemical results [Wang et al., 
2004], and has been demonstrated directly in slice experiments with intracellular recordings of Imc neurons coupled with 2-photon uncaging of glutamate [Goddard et al., 2014].

Nonetheless, because the role of this inhibition in vivo is not known, a direct test of the hypothesis that intra-Imc inhibition is the source of Imc's competitive responses will require inactivation of inhibitory projections between Imc neurons while recording their responses to the stimulus competition protocols that have been used here. Although one previous study has examined changes in the responses of Imc neurons following iontophoretic inactivation of synaptic inhibition onto them [Li et al., 2007], the results reported are difficult to interpret. This is because results from two different stimulus protocols appear to be combined: one, involving presentation of a single stimulus, and the other involving presentation of one stimulus inside and the other outside. As discussed above, because it is critical to consider results from these two protocols separately, the implications of that study to mechanistic underpinnings of Imc responses are not clear-cut. Consequently, the possibility of intra-Imc inhibition as the source of stimulus competition in the Imc remains to be verified.

An alternate explanation for the competitive responses observed in this study is that they do not reflect direct inhibition of Imc activity at all, but rather they reflect competitive interactions occurring in the OT, which are simply relayed back to the Imc. To appreciate this hypothesis, we consider below the anatomical and functional connectivity in the isthmo-tectal circuit (Fig. 6).

Anatomical studies have revealed that Imc neurons receive input exclusively from neurons in layer 10 of the OT $\left(\mathrm{OT}_{10}\right)$, but project back broadly across the space map in the OTid (layers 11-13) sparing just the portion of the map from which they receive input (Fig. 6) [Wang et al., 2004]. These neurons also project broadly to the nearby cholinergic isthmic nucleus called the Ipc. In parallel, Ipc neurons, which receive focal input (also) from neurons in $\mathrm{OT}_{10}$, project back focally to the space map of the OT (layers 1-13), innervating the same portion of the map from which it receives input [Wang et al., 2006]. Functional (inactivation) studies have confirmed that, consistent with this anatomical picture, Imc neurons generate competitive interactions across the OT and Ipc space maps [Marin et al., 2007; Mysore and Knudsen, 2013], whereas Ipc neurons serve as focal amplifiers of OT activity [Asadollahi and Knudsen, 2016].

In this context, if competitive inhibition from the Imc were to directly, or indirectly through Ipc feedback, af-

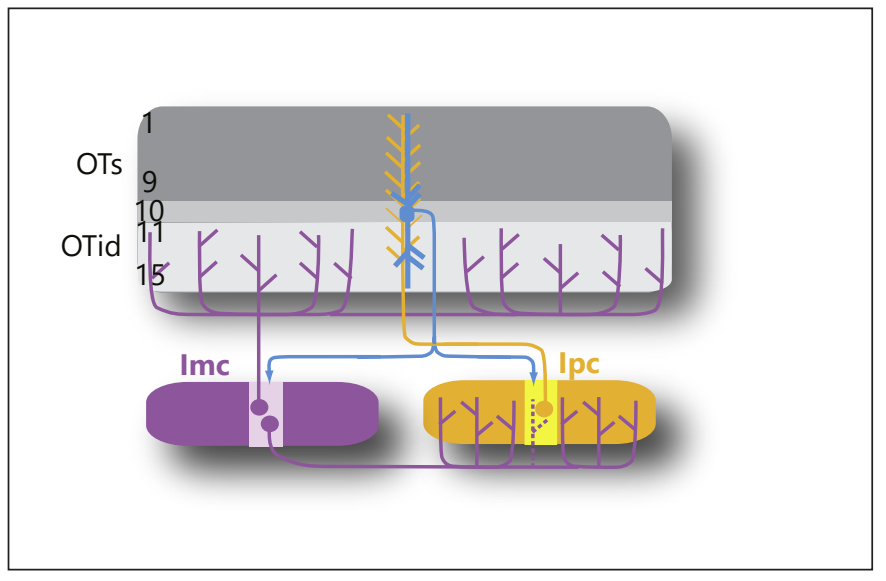

Fig. 6. Schematic of the anatomical connectivity of the isthmotectal circuit. Adapted from Wang et al. [2006]. Layers of the OT (in cross-sections), the GABAergic Imc, and the cholinergic Ipc are shown. The focal, topographic connectivity between Ipc and $\mathrm{OT}$ is shown $\left(\mathrm{OT}_{10} \rightarrow \mathrm{Ipc} \rightarrow \mathrm{OTs}\right)$, as well as the anti-topographic connectivity between the OT and Imc (OT $10 \rightarrow$ Imc $\rightarrow$ OTid). Notably, no evidence has been found for a synaptic relationship between Imc (or Ipc) and $\mathrm{OT}_{10}$ [Gruberg et al., 1994; Wang et al., 2006].

fect $\mathrm{OT}_{10}$ responses, then even without intra-Imc inhibition, Imc responses would appear suppressed by virtue of $\mathrm{OT}_{10}$ output transmitting these competitive representations to the Imc. Notably, it is not yet known whether isthmic nuclei project back to the OT neurons from which they receive input, in other words, to $\mathrm{OT}_{10}$ neurons. In fact, it has been suggested that isthmic neurons may not contact $\mathrm{OT}_{10}$ neurons [Gruberg et al., 1994; Wang et al., 2006]. If true, then the neurons that provide input to the Imc would potentially not be subject directly, or indirectly via Ipc, to inhibition from the Imc, thereby ruling out the idea that the Imc can "inherit" competitive responses. However, it is still possible that Imc neurons may influence $\mathrm{OT}_{10}$ activity through an even more indirect route, by effectively altering the retinal afferent input to $\mathrm{OT}_{10}$ [Wang et al., 2004; Wang et al., 2006].

Thus, the relative contributions of these "direct" versus "indirect" (respectively) hypotheses will need to be investigated explicitly in future work in order to unpack the mechanism(s) underlying Imc's responses.

Another puzzling mechanistic question is the source of the cross-hemispherical response suppression that was found here, and that has been reported in other components of the isthmo-tectal network [Asadollahi et al., 2010; Mysore et al., 2010]. In birds, Imc is known to project to the OT, Ipc, and itself, but only within the same 
hemisphere. Therefore, the circuit implementation of cross-hemispherical suppression must either be the result of direct interactions between the two OT hemispheres, or must involve forebrain structures as well. Future work will be needed to resolve this question.

\section{Sources of Inhibition Underlying Integration within} the $R F$

Work in the cortex and SC/OT offer the classic centersurround organization of RFs as a convincing explanation for response averaging within the RF. This relies on the existence of local inhibition, which is well known to exist both in the cortex and in the SC/OT, and is typically quantified with a difference-of-gaussians formulation. What is less clear is the source and implementation of local inhibition in the Imc - evidence to date does not support the presence of local inhibitory circuits within the Imc [Goddard et al., 2014). A plausible potential explanation, therefore, is that the Imc inherits response averaging from $\mathrm{OT}_{10}$ neurons.

Taken together, the results from this study expand our understanding of the functional properties of Imc neurons, specifically, their responses to two stimuli presented simultaneously, and represent a key step in exploring stimulus competition in the midbrain selection network. Exactly how these signatures of stimulus competition among distant stimuli (i.e., global inhibitory surround), and integration among nearby stimuli (i.e., classical inhibitory surround), are implemented in Imc's circuitry are intriguing open questions.

\section{Choice of Animal Model}

In this study, functional properties of the Imc, an inhibitory midbrain nucleus thought to be involved in the control of stimulus selection and spatial attention, were revealed with neurophysiological experiments in barn owls. Whereas mammals, and specifically monkeys, have historically been the animal of choice to study the neural basis of selective attention, birds, and specifically owls, offer a powerful benefit (discussed below), making them the animal model of choice for our experiments.

A study from 4 decades ago in reptiles first revealed a striking pattern of anatomical connectivity between the sensorimotor hub, the OT, and satellite nuclei in the midbrain isthmus [Sereno and Ulinski, 1987]. This same pattern was subsequently identified in birds and has been schematized in Figure 6: a group of magnocellular neurons, since shown to be GABAergic [Wang et al., 2004], receive focal input from the OT, but project back to the $\mathrm{OT}$ in a widespread manner sparing just the portion of the OT providing input [Wang et al., 2004]. Based on this pattern of connectivity, it was suggested that the Imc-OT circuit, conserved across all vertebrate classes [Knudsen, 2011], is potentially well-suited to serve as a control circuit for spatial selection [Sereno and Ulinski, 1987]. Combined with the demonstration of the critical role of the SC in the control of spatial attention [McPeek and Keller, 2004; Lovejoy and Krauzlis, 2010], a functional investigation of the role of the midbrain Imc in competitive selection emerged as an efficacious strategy to unpack explicit circuit mechanisms underlying the control of spatial selection and attention [Mysore and Knudsen, 2013].

Among all vertebrates, however, the midbrain circuitry of the OT and the isthmic nuclei are best elaborated in birds [Knudsen, 2011], providing a distinct experimental advantage in investigating the causal functions of the isthmic nuclei. Specifically, cholinergic and GABAergic neurons in the isthmus are anatomically well segregated, allowing for cell type-specific targeting of neural recordings and causal manipulations using stereotaxic approaches.

Among birds, owls offer a distinct advantage for these investigations because of their behavioral facility in more than one sensory modality: they are specialists in both the visual and auditory senses, making them well suited to investigate questions related to cross-modal selection, an integral facet of spatial attention.

Thus, inspired by a midbrain circuit hypothesis from reptiles for the control of spatial attention, the barn owl has emerged as an ideal model to dissect the functional logic of that midbrain circuit in a conceptually powerful (cross-modal) and experimentally tractable manner. Indeed, the choice of the barn owl for these investigations has proven fruitful previously [Asadollahi et al., 2010, 2011; Mysore et al., 2010; Mysore et al., 2011; Mysore and Knudsen, 2011a, 2012, 2013, 2014; Asadollahi and Knudsen, 2016; Mahajan and Mysore, 2018]. As evidenced by this study, the barn owl model continues to illuminate the workings of this important isthmotectal midbrain circuit in competitive stimulus selection and spatial attention.

\section{Acknowledgements}

We thank James Garmon for assistance in building the equipment necessary for the experiments. 


\section{Statement of Ethics}

All procedures for animal care and use were carried out following approval by the Johns Hopkins University Institutional Animal Care and Use Committee, and in accordance with NIH guidelines for the care and use of laboratory animals.

\section{Disclosure Statement}

The authors have no conflicts of interest to declare.

\section{Funding Sources}

This work was supported by funding from NIH grant R01EY027718.

\section{Author Contributions}

S.P.M. designed the study. H.M.S. and S.P.M. performed the experiments, analyzed the data, and wrote the paper.

\section{References}

Alvarado JC, Vaughan JW, Stanford TR, Stein BE. Multisensory versus unisensory integration: contrasting modes in the superior colliculus. J Neurophysiol. 2007 May;97(5):3193-205.

Asadollahi A, Knudsen EI. Spatially precise visual gain control mediated by a cholinergic circuit in the midbrain attention network. Nat Commun. 2016 Nov;7(1):13472.

Asadollahi A, Mysore SP, Knudsen EI. Stimulusdriven competition in a cholinergic midbrain nucleus. Nat Neurosci. 2010 Jul;13(7):88995.

Asadollahi A, Mysore SP, Knudsen EI. Rules of competitive stimulus selection in a cholinergic isthmic nucleus of the owl midbrain. J Neurosci. 2011 Apr;31(16):6088-97.

Bisley JW, Goldberg ME. Attention, intention, and priority in the parietal lobe. Annu Rev Neurosci. 2010;33(1):1-21.

Falkner AL, Krishna BS, Goldberg ME. Surround suppression sharpens the priority map in the lateral intraparietal area. J Neurosci. 2010 Sep; 30(38):12787-97.

Fecteau JH, Munoz DP. Salience, relevance, and firing: a priority map for target selection. Trends Cogn Sci. 2006 Aug;10(8):382-90.

Frost BJ. Moving background patterns alter directionally specific responses of pigeon tectal neurons. Brain Res. 1978 Aug;151(3):599603.

Goddard CA, Huguenard J, Knudsen E. Parallel midbrain microcircuits perform independent temporal transformations. J Neurosci. 2014 Jun;34(24):8130-8.

Goddard CA, Mysore SP, Bryant AS, Huguenard JR, Knudsen EI. Spatially reciprocal inhibition of inhibition within a stimulus selection network in the avian midbrain. PLoS One. 2014 Jan;9(1):e85865.

Gruberg ER, Hughes TE, Karten HJ. Synaptic interrelationships between the optic tectum and the ipsilateral nucleus isthmi in Rana pipiens. J Comp Neurol. 1994;339(3):353-64.

Knudsen EI. Auditory and visual maps of space in the optic tectum of the owl. J Neurosci. 1982; 2:1177-94.
Knudsen EI. Control from below: the role of a midbrain network in spatial attention. Eur J Neurosci. 2011;33(11):1961-72.

Krauzlis RJ, Lovejoy LP, Zénon A. Superior colliculus and visual spatial attention. Annu Rev Neurosci. 2013 Jul;36(1):165-82.

Li DP, Xiao Q, Wang SR. Feedforward construction of the receptive field and orientation selectivity of visual neurons in the pigeon. Cereb Cortex. 2007 Apr;17(4):885-93.

Li X, Basso MA. Competitive stimulus interactions within single response fields of superior colliculus neurons. J Neurosci. 2005;25(49): 11357-73.

Lovejoy LP, Krauzlis RJ. Inactivation of primate superior colliculus impairs covert selection of signals for perceptual judgments. Nat Neurosci. $2010 \mathrm{Feb} ; 13(2): 261-6$.

Mahajan NR, Mysore SP. Combinatorial neural inhibition for stimulus selection across space. Cell Rep. 2018;25(5):1158-70.e9.

Marín G, Salas C, Sentis E, Rojas X, Letelier JC, Mpodozis J. A cholinergic gating mechanism controlled by competitive interactions in the optic tectum of the pigeon. J Neurosci. 2007 Jul;27(30):8112-21.

McPeek RM, Keller EL. Deficits in saccade target selection after inactivation of superior colliculus. Nat Neurosci. 2004 Jul;7(7):757-63.

Mysore SP, Asadollahi A, Knudsen EI. Global inhibition and stimulus competition in the owl optic tectum. J Neurosci. 2010 Feb;30(5): 1727-38.

Mysore SP, Asadollahi A, Knudsen EI. Signaling of the strongest stimulus in the owl optic tectum. J Neurosci. 2011 Apr;31(14):5186-96.

Mysore SP, Knudsen EI. Flexible categorization of relative stimulus strength by the optic tectum. J Neurosci. 2011a May;31(21):7745-52.

Mysore SP, Knudsen EI. The role of a midbrain network in competitive stimulus selection. Curr Opin Neurobiol. 2011b Aug;21(4):65360.

Mysore SP, Knudsen EI. Reciprocal inhibition of inhibition: a circuit motif for flexible categorization in stimulus selection. Neuron. 2012 Jan;73(1):193-205.
Mysore SP, Knudsen EI. A shared inhibitory circuit for both exogenous and endogenous control of stimulus selection. Nat Neurosci. 2013 Apr;16(4):473-8.

Mysore SP, Knudsen EI. Descending control of neural bias and selectivity in a spatial attention network: rules and mechanisms. Neuron. 2014 Oct;84(1):214-26.

Reynolds JH, Chelazzi L, Desimone R. Competitive mechanisms subserve attention in macaque areas V2 and V4. J Neurosci. 1999 Mar; 19(5):1736-53.

Rizzolatti G, Camarda R, Grupp LA, Pisa M. Inhibitory effect of remote visual stimuli on visual responses of cat superior colliculus: spatial and temporal factors. J Neurophysiol. 1974 Nov;37(6):1262-75.

Sceniak MP, Ringach DL, Hawken MJ, Shapley R. Contrast's effect on spatial summation by macaque V1 neurons. Nat Neurosci. 1999 Aug; 2(8):733-9.

Sereno MI, Ulinski PS. Caudal topographic nucleus isthmi and the rostral nontopographic nucleus isthmi in the turtle, Pseudemys scripta. J Comp Neurol. 1987 Jul;261(3):319-46.

Squire RF, Noudoost B, Schafer RJ, Moore T. Prefrontal contributions to visual selective attention. Annu Rev Neurosci. 2013 Jul;36(1):45166.

Wang Y, Luksch H, Brecha NC, Karten HJ. Columnar projections from the cholinergic nucleus isthmi to the optic tectum in chicks (Gallus gallus): a possible substrate for synchronizing tectal channels. J Comp Neurol. 2006 Jan;494(1):7-35.

Wang Y, Major DE, Karten HJ. Morphology and connections of nucleus isthmi pars magnocellularis in chicks (Gallus gallus). J Comp Neurol. 2004 Feb;469(2):275-97.

Wang YC, Frost BJ. Visual response characteristics of neurons in the nucleus isthmi magnocellularis and nucleus isthmi parvocellularis of pigeons. Exp Brain Res. 1991;87(3):624-33.

Witten IB, Knudsen EI, Sompolinsky H. A Hebbian learning rule mediates asymmetric plasticity in aligning sensory representations. J Neurophysiol. 2008;100(2):1067-79. 